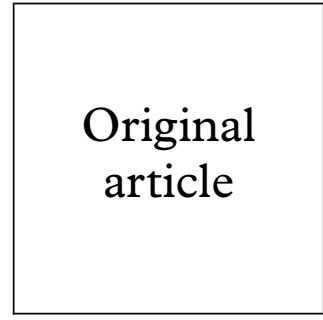

\section{Managing STIs identified after testing outside genitourinary medicine departments: one model of care}

\author{
L Haddon, J Heason, T Fay, M McPherson, E M Carlin, I H Ahmed Jushuf
}

\begin{abstract}
Objectives: To develop a local strategy for managing cases of Chlamydia trachomatis (CT) and Neisseria gonorrhoeae (GC) which have been identified in the departments of obstetrics and gynaecology (O\&G)

Methods: Weekly notification from the local microbiology laboratory to genitourinary medicine (GUM) departments of all positive CT and GC results generated by tests performed in the two local O\&G departments. Direct contact made by GUM departments to index patients identified and "fast track" appointments made. Data recorded for future audit include numbers attending, details of health adviser input, and success of contact tracing.

Results: Over 18 months, 294 women were identified and 231 (78\%) attended GUM departments; 142 (48\%) had received antibiotics before attending GUM departments and of these, $58(41 \%)$ had risked reinfection by an untreated partner and $48(20 \%)$ were found on screening to have a previously undiagnosed genital infection. Over $90 \%$ were interviewed by a health adviser. Appropriate follow up was achieved in $87 \%$ of index cases. Of the contacts, 194 were treated-150 in the local GUM department. Of these 150 men, 99(66\%) had an identifiable genital infection and $84 \%$ of those with $\mathrm{CT} /$ non-gonococcal urethritis were asymptomatic. There have been no complaints either formal or informal, by women managed by this system.

Conclusions: GUM clinics are the ideal setting to achieve successful treatment of patients with sexually acquired infections, which must include notification and treatment of their partners if reinfection is to be avoided. For patients with infections diagnosed in other settings, such as $\mathrm{O} \& \mathrm{G}$, a system of direct notification of results to GUM departments by an agreed protocol can be highly successful. For such a system to work, close cooperation and trust between departments is essential.
\end{abstract}

(Sex Transm Inf 1998;74:256-257)

Keywords: STIs; genitourinary medicine departments

\section{Introduction}

A recent Communicable Disease Report Supplement ${ }^{1}$ summarising the KC60 data sets for new cases seen at genitourinary medicine (GUM) clinics in England in 1995 and 1996 shows an overall increase of $20 \%$ for diagnoses of uncomplicated Neisseria gonorrhoeae (GC) and $11 \%$ for diagnoses of genital Chlamydia trachomatis (CT), with relatively large numbers of CT diagnosed in Trent. The incidence of the expensive sequelae of these infections is also increasing. In 1993 a regional audit in Trent showed disproportionately high rates of GC in Nottingham when compared with other districts such as Sheffield and Leicester. Nottingham also had high rates of the known sequelae of bacterial genital tract infections, such as pelvic inflammatory disease, ectopic pregnancy, and tubal infertility.

Women with sexually transmitted infections, and chlamydia in particular, may present in a variety of settings other than GUM clinics, including general practice, obstetrics and gynaecology (O\&G) departments, and family planning centres. During the year ending June 1993, 22 cases of GC and 58 cases of CT were identified in the local O\&G departments. These figures are significant when compared with the 408 cases of GC and 265 cases of CT identified in GUM departments during the same time period. The majority of the O\&G cases were not referred to GUM departments, and hence failed to benefit from GUM departments' expertise in health education, contact tracing, and follow up. In view of the apparent high levels of infection in the area, we decided to develop a mutually agreed, fail safe method of alerting women about their infection and encouraging attendance at GUM clinics.

\section{Method}

Once the need for improved access of O\&G patients to GUM departments had been ascertained, funding for increased health adviser work was required, for which we are indebted to Nottingham Health Authority. A mutually agreed protocol was introduced which enabled health advisers to contact all women with positive results (fig 1). As the backing of all O\&G consultants was essential for the smooth running of the project, many months were spent discussing possible pitfalls and seeking support for the project before implementation. We relied on the early enthusiasm of a number of the O\&G consultants to put the benefits of such a scheme to their colleagues. A pilot scheme was then implemented and the findings fed back after 6 months. 


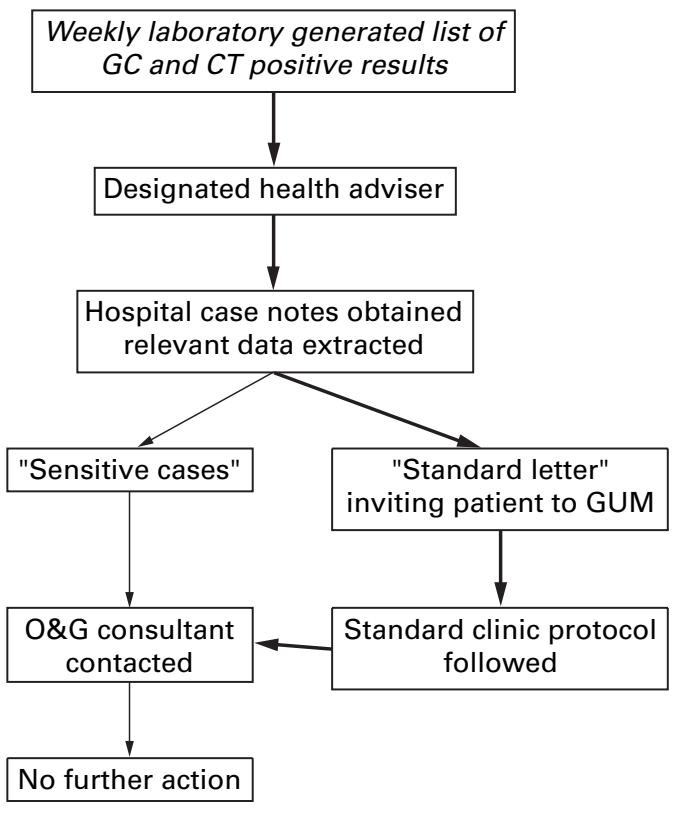

Figure 1 Patient care pathway.

\section{Results}

During the 18 month study period 294 women, mean age 22.1 years (range 14-45), were enrolled, having the following infections: CT 261, GC 13, dual CT, and GC 20. The most common reasons for testing were pretermination of pregnancy (107), during pregnancy or post partum (84), or for presumed pelvic inflammatory disease (80). All women were invited to the GUM department and 231 $(78.6 \%)$ attended, the majority within 2 weeks of invitation.

Before attending $142(48.3 \%)$ women had received antibiotics, and 58 of these $(41 \%)$ needed retreatment for possible reinfection. A previously undiagnosed genital infection was discovered in $48(20 \%)$ including GC five, Trichomonas vaginalis 14 , warts 19 , and herpes four. Over $90 \%$ were seen by a health adviser and appropriate follow up was achieved in 197 cases $(86.8 \%)$.

There was evidence that 194 contacts were treated as a direct result of this initiative- that is, of women attending GUM clinics, an average of $73.6 \%$ had one contact treated. Of these 150 were seen in Nottingham GUM department: 99 (66\%) had one or more genital infections, 77 had CT or non-gonococcal urethritis (NGU), 13 had GC, and five had dual infection. The majority $(84 \%)$ of those with $\mathrm{CT} / \mathrm{NGU}$ were asymptomatic.

\section{Discussion}

A significant proportion of chlamydial infection comes to light through tests performed in departments other than GUM. During the 18 month study period a total of 2178 positive CT tests were reported by the Nottingham laboratory, of which 1427 (66\%) were performed by GUM departments and $751(34 \%)$ were performed elsewhere. There has been a substantial increase in the number of CT tests performed outside GUM over the past 4 years (PHLS Nottingham, personal communica- tion). There may be important differences in the management of patients in different settings. Firstly, those diagnosed outside GUM departments would usually have no access to a health adviser or doctor experienced in contact tracing. A result of this might be a higher rate of reinfection due to coitus with an untreated partner. Experimental studies suggest that reinfection is more likely to lead to tubal damage than the original insult. ${ }^{2}$ Secondly, STI screening is routinely carried out in GUM clinics, and often reveals previously unsuspected conditions. Thirdly, management of the STI by GUM departments is more likely to be standardised and to follow recognised protocols than management elsewhere. ${ }^{34}$

Some reservations expressed by $O \& G$ departments at the time of introducing this protocol included a fear that their patients would be distressed at being invited to GUM departments, a view their patients were a different group of women from those attending GUM departments, and a sense that control of the patients' management would be lost. Our results clearly show that a high proportion of women $(78.6 \%)$ and their largely asymptomatic partners $(73.6 \%)$ attended GUM departments, indicating that GUM clinics were well accepted by this group of patients. Anxiety levels appeared to be no different from those of patients attending GUM departments directly, and many patient expressed their relief at having the diagnosis explained in a calm, nonjudgmental way. In order to allay any additional anxiety resulting from being contacted by GUM departments, the standard letter sent to the patient stated the name of the investigating O\&G consultant, thus emphasising the link between GUM and O\&G departments, and demonstrating the need for shared management. Once our action is complete in a case, a letter detailing treatment, follow up, and partner notification is sent to the relevant $O \& G$ consultant.

Effective management of STIs involves more than the use of correct antibiotics. Follow up, contact tracing and health education are also essential in every case. In addition, a sensitive supportive and informative approach is needed, with time and facilities to deal with patients' anxieties. In our opinion, widening the scope of STI testing to facilities outside GUM departments is to be welcomed. However, effective management is best achieved it this is coordinated with the local GUM department, where the essential components of effective STI management are routinely practised.

1 Simms I, Hughes G, Swan AV, et al. New cases seen at genitourinary medicine clinics: England 1996. CDR supplement 1998; 8:suppl 1.

2 Patton DL, Wolner-Hanssen P, Cosgrove SJ, et al. The effects of Chlamydia trachomatis on the female reproductive tract of the Macaca nemestrina after a single challenge and following repeated cervical inoculations. Obstet Gynecol 1990;76:643-50.

3 Reid S, Glucksman E, Blott M, et al. Do women with pelvic inflammatory disease receive adequate treatment? Int $f$ STD AIDS 1997;8:466-7.

4 Wales NM, Barton SE, Boag FC, et al. An audit of the management of pelvic inflammatory disease. Int $\mathcal{F} S T D$ AIDS agement of pelvic 